\title{
MODELING HIGH-CONCENTRATION PHOSPHORUS DIFFUSION IN CRYSTALLINE SILICON
}

\author{
O. I. Velichko, ${ }^{\text {a } V . ~ V . ~ A k s e n o v, ~}{ }^{\mathrm{b}}$ L. P. Anufriev, ${ }^{\mathrm{b}}$ \\ N. F. Golubev, ${ }^{b}$ and A. F. Komarov ${ }^{\mathrm{c}}$
}

UDC 539.219.3

Phosphorus diffusion in crystalline silicon in doping from a surface source at a temperature of $840^{\circ} \mathrm{C}$ and a duration of $10 \mathrm{~min}$ has been modeled. Good agreement with experimental data has been obtained, which confirms the adequacy of the employed model of high-concentration phosphorus diffusion. This model takes account of the drift of self-interstitials in the field of internal elastic stresses.

Keywords: phosphorus, silicon, diffusion, stresses, solar cell.

Introduction. Modeling of technological processes is widely used at present for development of new semiconductor devices. Of the processes used in the technology of creation of local doped regions of silicon devices, it is high-concentration phosphorus diffusion that stands out because of its most complicated behavior. Experimental data show that this diffusion has a number of distinctive features strongly differentiating it from the diffusion of other impurities of the elements of the Vth group. For example, the concentration profiles of phosphorus in diffusion from a source with a constant concentration of the impurity at the semiconductor surface have an "inflection" point on the phosphorus profile and the so-called "tail" in the region of low concentration of the impurity. With increase in the diffusion time the "inflection" point and the "tail" shift to the semiconductor volume, which gives rise to a "plateau" with a nearly constant concentration of the electrically active impurity at the substrate surface [1]. Another characteristic regularity of high-concentration phosphorus diffusion is the accelerated redistribution of boron, gallium, phosphorus, and arsenic in the already formed regions. In the technology of semiconductor devices, a consequence of this phenomenon is the dip effect of the base of $n-p-n$ transistors when the emitter is formed by high-concentration phosphorus diffusion. Acceleration of the diffusion of the impurity introduced earlier may attain significant values and manifest itself at a large distance from the phosphorus-doped region. Thus, from the data of [2], the diffusion coefficient of gallium in the buried layer was increased nearly 8 and 100 times compared to the thermally equilibrium layer, when the emitter was formed at temperatures of 1050 and $900^{\circ} \mathrm{C}$ respectively. The accelerated boron redistribution was investigated in [3]; it has been obtained that, at a temperature of $900^{\circ} \mathrm{C}$, the characteristic length of reduction of accelerated diffusion is equal to $30 \mu \mathrm{m}$, whereas the average increase in the diffusion coefficient of boron in a $40-\mu \mathrm{m}$ surface region is $\sim 100$ times. According to [3], the acceleration of the boron redistribution is strictly interrelated with the accelerated diffusion of phosphorus in the "tail" part of its distribution. Accelerated redistribution also occurs in the buried layer formed by the diffusion of the phosphorus itself [4]. A characteristic feature of the process of accelerated diffusion is the symmetry of the broadening of the profiles of the buried-layer impurity. This has been shown experimentally in $[2,3,5]$. However, the high-concentration phosphorus diffusion leads to a retardation of the redistribution of antimony atoms in the buried layer formed with the impurity [6]. A substantial influence of the process of phosphorus diffusion is exerted by the deformation of the crystal lattice due to the difference in the covalent radii of the impurity atoms and the silicon atoms. The above effects disappear or become much weaker with decrease in the degree of deformation of the lattice in the case of the codiffusion of phosphorus and germanium [7]. Thermal phosphorus diffusion leads to substantial changes in the fault subsystem of the crystals. Indeed, it has been shown in [8] that the processes of thermal diffusion of phosphorus, boron, or arsenic are responsible for the accelerated reduction in interstitial stacking faults in the region of occurrence of the impurity atoms. At the same time, it has been shown experimentally in $[6,9,10]$ that in high-concentration phosphorus diffusion, we have the supersaturation of the silicon volume with self-interstitials and the decrease in the concentration of vacancies, which produces a growth in the interstitial stacking faults beyond the region of high concentration of the impurity.

${ }^{a}$ Belarusian State University of Informatics and Radioelectronics, 6 P. Brovka Str., Minsk, 220013, Belarus; ${ }^{b}$ JointStock Company "Integral," 1 Kazinets Sq., Minsk, 220108, Belarus; ${ }^{c}$ Scientific-Research Institute "Sevchenko Institute of Applied Physical Problems," 7 Kurchatov Str., Minsk, 220045, Belarus; email: oleg_velichko@ cosmostv.by. Translated from Inzhenerno-Fizicheskii Zhurnal, Vol. 86, No. 3, pp. 626-634, May-June, 2013. Original article submitted December $18,2012$. 
To explain the distinctive features of the phosphorus profiles and other characteristics accompanying highconcentration doping, tens of phosphorus-diffusion models, have been developed [11-19]. These models can be subdivided into three groups according to the mechanisms of impurity transfer. The first group includes models in which the impurity transfer is assumed to be only through the migration of $\mathrm{E}$ centers, i.e., of the complexes of phosphorus atoms with a vacancy $[11,13,14]$. This mechanism of phosphorus diffusion is contradictory to the phenomenon of supersaturation of the semiconductor volume with silicon interstitials, which was pointed to in [6]. In the second group of models, one assumes that direct interstitial migration of nonequilibrium impurity interstitials occurs in addition to the traditional mechanisms of diffusion by means of E centers; this migration is responsible for the formation of "tails" [12]. These models also cannot explain the phenomenon of supersaturation of the silicon volume with self-interstitials and long-range effects of accelerated redistribution of the impurities introduced earlier. We emphasize that in the model of [12], an attempt was made to take account of the influence of the deformation of the crystal lattice on the process of phosphorus diffusion. Thus, it is assumed in [12] that deformation is the cause of the generation of phosphorus interstitials. Finally, in models of the third group, the transfer of phosphorus atoms is assumed to be through the formation of the complexes "phosphorus atom-silicon interstitial" [16-19]. Closely related to these works is the model in [15] where the transfer of phosphorus atoms is assumed to proceed by the relay mechanism of diffusion. Unlike the transfer mechanisms considered earlier, the mechanism of formation of mobile "phosphorus atom-silicon interstitial" complexes makes it possible to explain many of the regularities of the processes of high-concentration phosphorus diffusion. Thus, the assumption that the self-interstitial distribution is nonuniform makes it possible to explain the specific form of the distribution of phosphorus atoms on diffusion from a constant source [16, 17]. This nonuniform distribution can be formed both by the absorption of silicon interstitials in the highly-doped region [16] and as a consequence of their transfer beyond the doped region through the diffusion and disintegration of the complexes [17]. The assumption of the generation of nonequilibrium silicon interstitial makes it possible to explain the processes of accelerated diffusion of phosphorus atoms in the "tail" region and the accelerated impurity redistribution in the buried layers alike [16]. Also, the transfer of silicon interstitials through the diffusion and disintegration of the complexes makes it possible to explain the phenomenon of supersaturation of the semiconductor volume with self-interstitials. However, the models of the third group fail to explain the disappearance of the distinctive features of high-concentration phosphorus diffusion with decrease in internal elastic stresses in the case of phosphorus and germanium codiffusion. Furthermore, it is assumed in $[17,18]$ that the fluxes of the phosphorus atoms $j$ and of the silicon interstitials $j^{\mathrm{I}}$ are related by $j+j^{\mathrm{I}}=0$. This assumption is inconsistent with the description of the diffusion of nonequilibrium silicon interstitials in the semiconductor volume, since the concentration of the impurity there and its flux $j$ are equal to zero. Furthermore, it follows from the relation proposed that the concentration of the nonequilibrium silicon atoms is comparable with the concentration of the complexes. This seems unlikely, since the complexes are formed by the interaction of these atoms with the phosphorus atoms and it should be expected that the concentration of the complexes is lower in value than the concentration of phosphorus and the concentration of the self-interstitials alike.

The phosphorus-diffusion model in $[20,21]$ is free from the limitations described. According to this model, the transfer of phosphorus atoms proceeds by the two-flux mechanism of impurity diffusion through the formation, migration, and disintegration of the complexes "phosphorus atom-vacancy" and "phosphorus atom-silicon interstitial"; these complexes are in local thermodynamic equilibrium with the impurity atoms in the substitutional position and with the point defects forming these complexes, i.e., with the vacancies and self-interstitials, respectively. Point defects in various charge states are assumed to be involved in the complexation. Account taken of the nonuniform distribution of the silicon interstitials in a neutral charge state enables us to explain the distinctive features of the shape of the phosphorus atomic profile, namely, the formation of the "inflection" point and of the "tail" in the region of low concentration of the impurity [16]. It is assumed in the model of [20, 21] that the nonuniform distribution of the silicon interstitials is formed by the action of internal elastic stresses due to the difference in the covalent radii of the phosphorus $(0.110 \mathrm{~nm})$ and silicon $(0.117 \mathrm{~nm})$ atoms [22]. As follows from the given values, a dramatic compression of the crystal lattice will be observed in the region of the doped layer in high-concentration phosphorus diffusion. The drift of the nonequilibrium silicon interstitials in the field of elastic stresses beyond the phosphorus-doped layer leads to supersaturation of the semiconductor volume with self-interstitials and to an accelerated diffusion of impurity atoms in the buried layers. The disappearance of the distinctive features of high-concentration phosphorus diffusion in the case of the codiffusion of phosphorus and germanium is easily explained then by the decrease in the internal elastic stresses due to the larger covalent atomic radius of germanium $(0.122 \mathrm{~nm})$ compared to the radius of silicon [22].

It should be noted that the processes of high-concentration phosphorus diffusion have attracted close attention recent because of their use for the creation of doped regions in solar cells based on crystalline silicon [23-25] and for 
gettering of undesirable iron-type impurities [26, 27]. In this connection, it makes sense to apply the model developed to the investigation of recent experimental data on high-concentration phosphorus diffusion in manufacturing solar cells.

Equations of the Phosphorus-Diffusion Model. To describe the processes of phosphorus diffusion at a high concentration of impurity atoms, we employ the following system of equations:

(1) the equation of phosphorus atomic diffusion, which takes account of the drift of the complexes "phosphorus atom-vacancy" and "phosphorus atom-silicon interstitial" in the field of internal elastic stresses [28]:

$$
\begin{aligned}
C_{t}^{T} & =\frac{\partial}{\partial x}\left[D^{\mathrm{E}} \frac{\partial\left(a^{\mathrm{E}} \tilde{C}^{\mathrm{V} \times} C\right)}{\partial x}+\frac{D^{\mathrm{E}} a^{\mathrm{E}} \tilde{C}^{\mathrm{V} \times} C}{\chi} \frac{\partial \chi}{\partial x}\right]-\frac{\partial\left(\mathbf{v}^{\mathrm{E}} a^{\mathrm{E}} \tilde{C}^{\mathrm{V} \times} C\right)}{\partial x} \\
& +\frac{\partial}{\partial x}\left[D^{\mathrm{F}} \frac{\partial\left(a^{\mathrm{F}} \tilde{C}^{\mathrm{I} \times} C\right)}{\partial x}+\frac{D^{\mathrm{F}} a^{\mathrm{F}} \tilde{C}^{\mathrm{I} \times} C}{\chi} \frac{\partial \chi}{\partial x}\right]-\frac{\partial\left(\mathbf{v}^{\mathrm{F}} a^{\mathrm{F}} \tilde{C}^{\mathrm{I} \times} C\right)}{\partial x},
\end{aligned}
$$

where

$$
\begin{gathered}
D^{\mathrm{E}}=D_{i}^{\mathrm{E}} D^{\mathrm{EC}}(\chi), D^{\mathrm{EC}}(\chi)=\frac{1+\beta_{1}^{\mathrm{E}} \chi+\beta_{2}^{\mathrm{E}} \chi^{2}}{1+\beta_{1}^{\mathrm{E}}+\beta_{2}^{\mathrm{E}}}, D_{i}^{\mathrm{E}}=D_{i}^{\mathrm{E} \times}+D_{i}^{\mathrm{E} 1}+D_{i}^{\mathrm{E} 2}, \beta_{1}^{\mathrm{E}}=\frac{D_{i}^{\mathrm{E} 1}}{D_{i}^{\mathrm{E} \times}}, \\
\beta_{2}^{\mathrm{E}}=\frac{D_{i}^{\mathrm{E} 2}}{D_{i}^{\mathrm{E} \times}}, D^{\mathrm{F}}=D_{i}^{\mathrm{F}} D^{\mathrm{FC}}(\chi), D^{\mathrm{FC}}(\chi)=\frac{1+\beta_{1}^{\mathrm{F}} \chi}{1+\beta_{1}^{\mathrm{F}}}, D_{i}^{\mathrm{F}}=D_{i}^{\mathrm{F} \times}+D_{i}^{\mathrm{F} 1}, \beta_{1}^{\mathrm{F}}=\frac{D_{i}^{\mathrm{F} 1}}{D_{1}^{\mathrm{F} \times}}, \\
\tilde{C}^{\mathrm{V} \times}=C^{\mathrm{V} \times} / C_{\mathrm{eq}}^{\mathrm{V} \times}, \tilde{C}^{\mathrm{I} \times}=C^{\mathrm{I} \times} / C_{\mathrm{eq}}^{\mathrm{I} \times} .
\end{gathered}
$$

(2) the approximation of local electric neutrality for the reduced concentration of charge carriers $\chi=n / n_{i}$ :

$$
\chi=\frac{C-N^{\mathrm{B}}+\sqrt{\left(C-N^{\mathrm{B}}\right)^{2}+4 n_{i}}}{2 n_{i}}
$$

(3) the equation of silicon interstitial diffusion, which will be represented as [20,21]:

$$
\frac{\partial}{\partial x}\left[d^{\mathrm{IC}}(\chi) \frac{\partial \tilde{C}^{\mathrm{I} \times}}{\partial x}\right]-\frac{\partial}{\partial x}\left(\frac{\mathbf{v}^{\mathrm{I}}}{d_{i}^{\mathrm{I}}} \tilde{C}^{\mathrm{I} \times}\right)-\frac{k^{\mathrm{IC}}(x, \chi) \tilde{C}^{\mathrm{I} \times}}{\left(l_{i}^{\mathrm{I}}\right)^{2}}+\frac{\tilde{g}^{\mathrm{I}}}{\left(l_{i}^{\mathrm{I}}\right)^{2}}=0,
$$

where

$$
d^{\mathrm{IC}}(\chi)=\frac{d^{\mathrm{I}}(\chi)}{d_{i}^{\mathrm{I}}}, l_{i}^{\mathrm{I}}=\sqrt{d_{i}^{\mathrm{I}} \tau_{i}^{\mathrm{I}}}, k^{\mathrm{IC}}(x, \chi)=\left(\frac{\tau^{\mathrm{I}}}{\tau_{i}^{\mathrm{I}}}\right)^{-1}, \tilde{g}^{\mathrm{I}}=\frac{g^{\mathrm{I}} \tau_{i}^{\mathrm{I}}}{C_{\mathrm{eq}}^{\mathrm{I} \times}}=\frac{g^{\mathrm{I}}}{g_{i}^{\mathrm{I}}} .
$$

(4) the equation of vacancy diffusion, which will be represented as [20, 21]:

$$
\begin{gathered}
\frac{\partial}{\partial x}\left[d^{\mathrm{VC}}(\chi) \frac{\partial \tilde{C}^{\mathrm{V} \times}}{\partial x}\right]-\frac{\partial}{\partial x}\left(\frac{\mathbf{v}^{\mathrm{V}}}{d_{i}^{\mathrm{V}}} \tilde{C}^{\mathrm{V} \times}\right)-\frac{k^{\mathrm{VC}}(x, \chi) \tilde{C}^{\mathrm{V} \times}}{\left(l_{i}^{\mathrm{V}}\right)^{2}}+\frac{\tilde{g}^{\mathrm{V}}}{\left(l_{i}^{\mathrm{V}}\right)^{2}}=0, \\
d^{\mathrm{VC}}(\chi)=\frac{d^{\mathrm{V}}(\chi)}{d_{i}^{\mathrm{V}}}, l_{i}^{\mathrm{V}}=\sqrt{d_{i}^{\mathrm{V}} \tau_{i}^{\mathrm{V}}}, k^{\mathrm{VC}}(x, \chi)=\left(\frac{\tau^{\mathrm{V}}}{\tau_{i}^{\mathrm{V}}}\right)^{-1}, \tilde{g}^{\mathrm{V}}=\frac{g^{\mathrm{V}} \tau_{i}^{\mathrm{V}}}{C_{\mathrm{eq}}^{\mathrm{V} \times}}=\frac{g^{\mathrm{V}}}{g_{i}^{\mathrm{V}}} .
\end{gathered}
$$

From the above values of the acceleration of the impurity diffusion in the semiconductor volume, it follows that the concentration of nonequilibrium silicon interstitials beyond the phosphorus-doped layer exceeds many times the thermally equilibrium concentration of these particles. This means that in the phosphorus-doped layer, we have the generation of silicon interstitials. For example, such generation may occur when phosphorus atoms are built into the crystal lattice of silicon, as 
has been assumed in [6]. Furthermore, at a high concentration of the impurity, precipitates of silicon phosphoride SiP [29] or clusters of phosphorus atoms are formed [30]. The occurring quasichemical reactions may be accompanied by the generation of silicon interstitials. In turn the generated silicon interstitials find themselves in the elastic-stress field, which ensure their transfer beyond the region of high concentration of phosphorus. To find the distribution of the effective drift velocity of the silicon interstitials, we must calculate the distribution of the internal elastic stresses in the doped layer. However, the distribution of these stresses depends not only on the distribution of the phosphorus atoms but also on the distribution of other components of the fault subsystem of the crystal, namely, on the vacancies, silicon interstitials, clusters, or precipitates of phosphorus atoms. The solution of this problem is extremely difficult and is not the aim of the present work. Therefore, we approximate the distribution of the effective drift velocity of silicon interstitials by the following semiempirical dependence:

$$
\mathbf{v}^{\mathrm{I}}(x)= \begin{cases}\mathbf{v}_{\text {max }}^{\mathrm{I}}, & x<x^{*}, \\ \mathbf{v}_{\max }^{\mathrm{I}} \exp \left[-\frac{\left(x-x^{*}\right)}{2 \Delta R_{\mathrm{p} . \mathrm{st}}^{2}}\right], & x \geq x^{*} .\end{cases}
$$

The parameter $x^{*}$ characterizes the width of the range of action of the internal elastic stresses and may be related to the characteristic value of the impurity concentration $C^{*}$, which determines the position of the inflection point on the phosphorus profile.

We approximate the rate of generation of the nonequilibrium silicon interstitials by an analogous dependence:

$$
\tilde{g}^{\mathrm{I}}(x)= \begin{cases}1+\tilde{g}_{\max }^{\mathrm{I}}, & x<x^{*}, \\ 1+\tilde{g}_{\max }^{\mathrm{I}} \exp \left[-\frac{\left(x-x^{*}\right)}{2 \Delta R_{\mathrm{p.d}}^{2}}\right], & x \geq x^{*} .\end{cases}
$$

The proposed distribution of the generation rate of the silicon interstitials (6) is close to the distribution of the phosphorus atoms in the region of high concentration of the impurity, as is the distribution (5). This confirms our assumption that the distributions (5) and (6) are fairly good approximations for description of poorly studied processes of generation of nonequilibrium faults and of deformation of the crystal lattice. Since for the experimental data in question, the process of clustering exists in an exclusively narrow surface region, for the approximate description of this process and for calculation of the quantity $C^{\mathrm{AC}}$, we have used the standard expression

$$
\frac{C^{\mathrm{AC}}}{C^{\mathrm{AC}}}=\left(\frac{C}{C^{\mathrm{AC}}}\right)^{m}
$$

The quantity $m$ in expression (7) is set equal to 3, which corresponds to the dependence of [11] for the concentration of electrically inactive phosphorus.

Results of Numerical Calculations. The results of modeling the process of high-concentration phosphorus diffusion, which have been obtained with the model proposed, are given in Fig. 1. Numerical solution of the system of transfer equations (1), (3), and (4) for the one-dimensional case in question was carried out by the finite-difference method [31]. The data of [23] were used to compare calculation results and experiment. In [23], high-concentration phosphorus doping was carried out using phosphosilicate glass formed on the surface of a silicon substrate on its treatment in a $\mathrm{POCl}_{3}, \mathrm{O}_{2}$, and $\mathrm{N}_{2}$ atmosphere at a temperature of $840^{\circ} \mathrm{C}$ for $10 \mathrm{~min}$. This glass represents a mixture of phosphorus pentoxide $\left(\mathrm{P}_{2} \mathrm{O}_{5}\right)$ and silica $\left(\mathrm{SiO}_{2}\right)$ and may be considered, according to the data of [23], as a diffusion source with a constant concentration of the impurity of the semiconductor surface. In the experiment, we used silicon substrates (grown by the method of a floating $p$-conductivity zone) doped with boron with a resistivity of $2 \Omega \cdot \mathrm{cm}$. The profile of electrically active phosphorus was measured by the electrochemical volt-farad method [23]. The measured profile of phosphorus atoms in the substitutional position is also presented in Fig. 1.

In modeling the high-concentration phosphorus diffusion, we used the following values of the parameters of the process of transfer of impurity atoms and intrinsic point defects. The intrinsic concentration of the charge carriers was $n_{i} \approx 3.213$ $\times 10^{6} \mu \mathrm{m}^{-3}$; the limit of solubility of phosphorus in silicon was $3.9 \times 10^{8} \mu \mathrm{m}^{-3}$ [32]. The intrinsic coefficient of phosphorus 


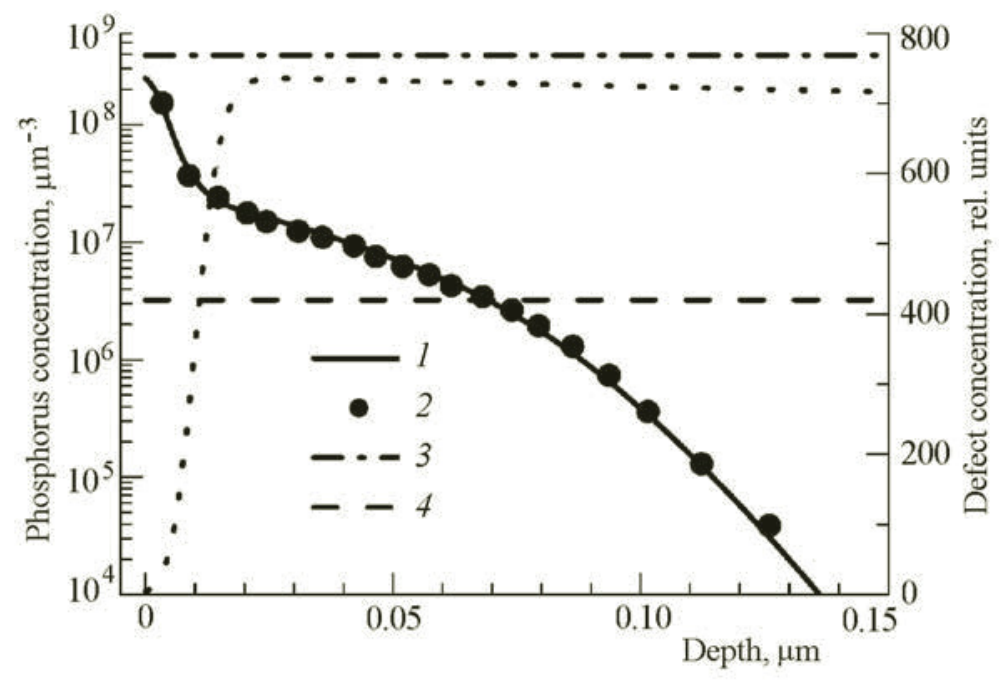

Fig. 1. Calculated phosphorus profiles and concentration distributions of silicon interstitials in the case of high-concentration diffusion from a constant source at $840^{\circ} \mathrm{C}$ for 10 min: 1) calculated distributions of phosphorus atoms after the diffusion; 2) experiment according to [23]; 3) limit of solubility of phosphorus in silicon; 4) concentration of intrinsic charge carriers. Dashed curve, concentration of silicon interstitials in a neutral charge state at the end of thermal treatment, normalized to the thermally equilibrium concentration of these particles.

diffusion $D_{i}=D_{i}^{\times}+D_{i}^{1}+D_{i}^{2}=2.047 \times 10^{-8} \mu \mathrm{m}^{2} / \mathrm{s}$, and the parameters describing the concentration dependence of the diffusion coefficient $\beta_{1}=D_{i}^{1} / D_{i}^{\times}=0.00035$ and $\beta_{2}=D_{i}^{2} / D_{i}^{\times}=0.008$ were taken from [33]. The value of the relative contribution of the indirect interstitial mechanism of transfer of impurity atoms in low-concentration diffusion $f_{i}=D_{i}^{\mathrm{F}} / D_{i}=0.084$ was taken from [34], which gives the values $D_{i}^{\mathrm{E}}=1.875 \times 10^{-8} \mu \mathrm{m}^{2} / \mathrm{s}$ and $D_{i}^{\mathrm{F}}=0.172 \times 10^{-8} \mu \mathrm{m}^{2} / \mathrm{s}$ (total value $D_{i}=D_{i}^{\mathrm{F}}+D_{i}^{\mathrm{E}}$ ). Because of the shortage of experimental data on diffusion by means of the complexes "phosphorus atom-self-interstitial," in the present work we assume the absence of the concentration dependence for the indirect mechanism of interstitial diffusion. Therefore, the above values of $\beta_{1}$ and $\beta_{2}$ were recalculated with account taken of the contribution of the indirect interstitial diffusion mechanism to the corresponding values $\beta_{1}^{\mathrm{E}}=0.00038$ and $\beta_{2}^{\mathrm{E}}=0.0087$ describing the concentration dependence for diffusion by means of vacancy-impurity complexes in the presence of the channel of interstitial diffusion by means of equilibrium complexes with a constant diffusion coefficient. The value of the diffusion mean free path of silicon interstitials $l_{i}^{\mathrm{I}}=30 \mu \mathrm{m}$ was selected in accordance with experimental data on the decrease in the acceleration of diffusion deep into the semiconductor [3]. At the same time, a few values of the parameters of the diffusion process were determined from the condition of the best agreement between the calculated concentration distribution of phosphorus and experimental data: the concentration of electrically active phosphorus atoms at the semiconductor surface $C_{\mathrm{s}}=2.49 \times 10^{8} \mu \mathrm{m}^{-3}$, the normalized rate of generation of nonequilibrium silicon interstitials $\tilde{g}_{\max }^{\mathrm{I}}=1.4 \times 10^{7}$ rel. units, the maximum value of the effective drift velocity of silicon interstitials $\mathbf{v}_{\max }^{\mathrm{I}} / d_{i}^{\mathrm{I}}=600.0 \mu \mathrm{m}^{-1}$, and $\Delta R_{\mathrm{p} . \mathrm{st}}=\Delta R_{\mathrm{p} . \mathrm{d}}=0.0067 \mu \mathrm{m}$.

As is seen from Fig. 1, the concentration of electrically active phosphorus is much lower than the limit of phosphorus solubility in silicon that has been calculated according to the data of [32]. Therefore, in the considered case of diffusion the clustering of impurity atoms is either totally absent or occurs in an exclusively narrow layer on the boundary with phosphosilicate glass, i.e., in the region situation with a maximum concentration of phosphorus. This means that it is quite difficult to obtain a certain value of the quantity $C^{\mathrm{AC}}$ from the results of fitting to experimental data. We have an analogous situation with determining a specific value of $C^{*}$. Indeed, we can only establish from the fitting results that the concentration $C^{*}$ is high enough for the characteristic depth $x^{*}$ of the range of action of elastic stresses to be nearly zero.

To obtain a numerical solution of the equation of phosphorus atomic diffusion we used 1001 spatial-discretization modes and 800 layers on the time axis. In modeling the process of phosphorus diffusion, by virtue of the shortage of experimental data, we neglected the drift of the complexes "impurity atom-intrinsic point defect" in the field of internal elastic stresses and the concentration dependence of the coefficient of diffusion of vacancies and silicon interstitials. 
Analysis of the Obtained Results. It is clear from Fig. 1 that the numerical calculation of high-concentration phosphorus diffusion, carried out with the model of $[20,21]$, is in good agreement with experimental data. The model developed in [20,21], in addition to being in quantitative agreement with experiment, enables us to explain the basic qualitative regularities of high-concentration phosphorus diffusion. Thus, unlike other models, it takes account of the influence, on the process of phosphorus diffusion, of the occurring deformation of the silicon crystal lattice. Moreover, account taken of the drift of silicon interstitials in the field of internal elastic stresses and the resulting nonuniform distribution of self-interstitials are the basic propositions of the model described. It is precisely from these propositions that we can explain substantial variations of the processes of diffusion of phosphorus from the diffusion of other impurities. For example, simultaneous doping of silicon with germanium atoms leads to a reduction in the deformation of the crystal lattice. In this case elastic stresses decrease in value and the distribution of self-interstitials becomes more uniform. Accordingly, qualitative differences between the process of high-concentration diffusion of phosphorus and the diffusion of other impurities decrease or totally disappear.

As is seen from the calculation presented in the figure, the concentration of silicon interstitials in the semiconductor volume exceeds the thermally equilibrium concentration approximately 730 times, i.e., the intensity of impurity transfer through the formation of the complexes "phosphorus atom-self-interstitial" in the region of formation of the "tail" grows an identical number of times. With account taken of the value of the contribution of the indirect interstitial mechanism, which has been used in calculating, this means that a nearly 62 -fold acceleration of the process of diffusion of phosphorus atoms occurs in the semiconductor volume compared to the thermally equilibrium low-concentration diffusion. It should be noted that the presented calculation of phosphorus diffusion was carried out under the assumption of the dominant role of the process of migration of vacancy-impurity complexes in the case of a low concentration of the impurity where there are no characteristic features of high-concentration doping. This assumption is consistent with the concentration dependence of the diffusion coefficient [33], since the presence of the term, which is proportional to the electron concentration squared, is easily explained by the formation of complexes of phosphorus with doubly negatively charged vacancies. Nonetheless, many works (e.g., [35-39]) have pointed to the dominant role of the indirect interstitial transfer mechanism, within whose framework one assumes the interaction of silicon interstitials with phosphorus atoms in the substitutional position, under any diffusion conditions; the share of this diffusion mechanism is close to $100 \%$. If this assumption is true, calculation of the process of high-concentration phosphorus diffusion would be analogous to the calculation in Fig. 1; only the concentrations of nonequilibrium silicon interstitials would decrease by approximately an order of magnitude. The value of the acceleration of the diffusion of impurity atoms in the region of formation of the "tail" and in the semiconductor volume would remain the same.

Conclusions. With the model of high-concentration phosphorus diffusion developed earlier [20,21], we have calculated the process of doping of silicon from phosphosilicate glass applied to the substrate surface at a temperature of $840^{\circ} \mathrm{C}$ and a duration of $10 \mathrm{~min}$. The process of transfer of phosphorus atoms, modeled in the present work, is used for creation of an $n$-conductivity region in manufacturing solar cells on crystalline silicon [23]. The results of numerical calculations of phosphorus diffusion from a constant source for which the phosphosilicate glass is used are in good agreement with experimental data. This confirms the adequacy of the employed model of transfer processes, within whose framework it is assumed that the distinctive features of high-concentration phosphorus diffusion are caused by the formation of a nonuniform distribution of silicon interstitials which are in a neutral charge state. The formation of this nonuniform distribution is due to the drift of self-interstitials in the field of internal elastic stresses that occur in the doped layer because of the difference in the atomic radii of phosphorus and silicon. As is seen from the calculations carried out, the drift of interstitials in the elastic-stress field leads to a considerable growth in the concentration of these particles in the semiconductor volume compared to the thermally equilibrium concentration, which enables us to explain the formation of the "tail" part on the phosphorus profile and the phenomenon of accelerated diffusion of boron, arsenic, and gallium in deep-lying buried layers.

\section{NOTATION}

$a^{\mathrm{E}}$, coefficient allowing for the deviation of the constants of local thermodymanic equilibrium at high doping levels of silicon; $a^{\mathrm{F}}$, coefficient allowing for the deviation of the constants of local thermodymanic equilibrium at high doping levels; $C^{\mathrm{AC}}$, concentration above which intense clustering occurs; $\tilde{C}^{\mathrm{V} \times}$ and $\tilde{C}^{\mathrm{I} \times}$, concentrations of vacancies and silicon interstitials in a neutral charge state, normalized to the thermally equilibrium concentrations of these particles $C_{\mathrm{eq}}^{\mathrm{V} \times}$ and $C_{\mathrm{eq}}^{\mathrm{I} \times}$ respectively; $C^{T}$ and $C$, total concentration of phosphorus and concentration of phosphorus atoms in the substitutional position; $d^{\mathrm{I}}(\chi)$ and $d_{i}^{\mathrm{I}}$, effective coefficient of diffusion of silicon interstitials and its value in the intrinsic semiconductor respec- 
tively; $d^{\mathrm{V}}(\chi)$ and $d_{i}^{\mathrm{V}}$, effective coefficient of diffusion of vacancies and its value in the intrinsic semiconductor respectively; $D^{\mathrm{E}}$ and $\mathbf{v}^{\mathrm{E}}$, respectively effective diffusion coefficient and effective velocity of drift of impurity atoms in the stress field by the mechanism of formation of vacancy-impurity complexes; $D_{i}^{\mathrm{E}}$, intrinsic coefficient of diffusion of phosphorus atoms by the above-indicated mechanism; $D_{i}^{\mathrm{Ex}}, D_{i}^{\mathrm{E} 1}$, and $D_{i}^{\mathrm{E} 2}$, partial coefficients of diffusion of the impurity using neutral and singly and doubly charged vacancies respectively; $D_{i}^{\mathrm{F}}$, intrinsic coefficient of diffusion of phosphorus atoms by the aboveindicated mechanism; $D_{i}^{\mathrm{F} \times}$ and $D_{i}^{\mathrm{F} 1}$, partial coefficients of diffusion of the impurity using neutral and singly charged silicon interstitials respectively; $D^{\mathrm{F}}$ and $\mathbf{v}^{\mathrm{F}}$, effective diffusion coefficient and effective drift velocity of impurity atoms in the stress field by the mechanism of formation of complexes with silicon interstitials respectively; $\tilde{g}_{\text {max }}^{I}$, maximum value of the normalized rate of generation of nonequilibrium silicon interstitials; $g^{\mathrm{I}}$ and $g_{i}^{\mathrm{I}}$, rate of generation of silicon interstitials in a unit volume of the semiconductor and rate of thermal generation of these particles in the intrinsic semiconductor respectively; $g^{\mathrm{V}}$ and $g_{i}^{\mathrm{V}}$, rate of generation of vacancies in a unit volume of the semiconductor and rate of thermal generation of vacancies in the intrinsic semiconductor respectively; $l_{i}^{\mathrm{V}}$, diffusion mean free path of vacancies in the intrinsic semiconductor; $l_{i}^{\mathrm{I}}$, diffusion mean free path of silicon interstitials in the intrinsic semiconductor; $N^{\mathrm{B}}$, concentration of acceptors; $\mathbf{v}_{\max }^{\mathrm{I}}$, maximum value of the drift velocity of silicon interstitials; $\mathbf{v}^{\mathrm{V}}$, effective drift velocity of vacancies in the field of internal elastic stresses; $\mathbf{v}^{\mathrm{I}}$, effective drift velocity of silicon interstitials in the stress field; $x^{*}$, characteristic dimension of the stress region; $\beta_{1}^{\mathrm{E}}$ and $\beta_{2}^{\mathrm{E}}$, parameters describing the relative contribution of singly and doubly charged vacancies respectively to the process of impurity transfer; $\beta_{1}^{\mathrm{E}}$, parameter describing the relative contribution of singly charged silicon interstitials to the process of impurity transfer; $\Delta R_{\mathrm{p} . \mathrm{st}}^{2}$, variance of the distribution of the drift velocity of particles; $\Delta R_{\mathrm{p} . \mathrm{d}}^{2}$, variance of the distribution of the generation rate of these particles; $\tau^{\mathrm{I}}$ and $\tau_{i}^{\mathrm{I}}$, mean lifetime of silicon interstitials and its value in the intrinsic semiconductor respectively; $\tau^{\mathrm{V}}$ and $\tau_{i}^{\mathrm{V}}$, mean lifetime of vacancies and its value in the intrinsic semiconductor respectively. Subscripts and superscripts: $\mathrm{t}$, total; V, vacancy; I, silicon interstitial; $i$, intrinsic; eq, equilibrium; max, maximum; p.st, projective for stresses; p.d, projective for defects.

\section{REFERENCES}

1. V. A. Panteleev, T. S. Gugina, and N. E. Rudoi, Anomalies of diffusion distribution of phosphorus in silicon, Fiz. Poluprovodn. Poluprovodn. Élektron., No. 8, 41-47 (1977).

2. C. L. Jones and A. F. W. Willoughby, Studies of the push-out effect in silicon: II. The effect of phosphorus emitter diffusion of gallium-base profiles determined by radiotracer techniques, J. Electrochem. Soc., 123, No. 10, 1531-1538 (1976).

3. D. Lecrosnier, M. Gauneau, J. Paugam, et al., Long-range enhancement of boron diffusivity induced by a high surfaceconcentration phosphorus diffusion, Appl. Phys. Lett., 34, No. 3, 224-226 (1979).

4. S. Matsumoto, E. Arai, H. Nakamura, and T. Niimi, The distribution of excess vacancies in the bulk at the diffusion of phosphorus into silicon, Jpn. J. Appl. Phys., 16, No. 7, 1177-1185 (1977).

5. D. V. Lee and A. F. W. Willoughby, A new observation of enhanced diffusion, J. Appl. Phys., 43, No. 1, 245-247 (1972).

6. P. Faney, R. W. Dutton, and S. M. Hu, Supersaturation of self-interstitials and undersaturation of vacancies during phosphorus diffusion in silicon, Appl.Phys.Lett., 44, No. 8, 777-779 (1984).

7. L. A. Koledov and L. N. Sergeev (V. S. Zemskii Ed.), Properties of Doped Semiconductors [in Russian], Nauka, Moscow (1977), pp. 143-145.

8. H. Hashimoto, H. Shibayama, H. Masaki, and H. Ishikawa, Annihilation of stacking faults in silicon by impurity diffusion, J. Electrochem. Soc., 123, No. 12, 1899-1902 (1976).

9. H. Strunk, U. Gosele, and B. O. Kolbesen, Interstitial supersaturation near-phosphorus diffused emitter zones in silicon, Appl. Phys. Lett., 34, No. 8, 530-532 (1979).

10. U. Gosele and H. Strunk, High-temperature diffusion of phosphorus and boron in silicon via vacancies or via selfinterstitials, Appl. Phys. A, 20, No. 4, 265-273 (1979).

11. R. B. Fair and J. C. C. Tsai, Quantative model for diffusion of phosphorus in silicon and emitter dip effect, $J$. Electrochem. Soc., 124, No. 7, 1107-1118 (1977).

12. V. A. Panteleev, Mechanism underlying the diffusion of phosphorus in silicon, Fiz. Tverd. Tela, 21, Issue11, 3388-3392 (1979). 
13. M. Yoshida, General theory of phosphorus and arsenic diffusion in silicon, Jpn. J. Appl. Phys., 19, No. 12, 2427-2440 (1980).

14. D. Mathiot and J. C. Prister, On the diffusion of donors into silicon: high concentration and nonequilibrium defect effects, Physica, BC-116, Nos. 1-3, 95-100 (1983).

15. H. F. Schaake, The diffusion of phosphorus in silicon from high surface concentration, J. Appl. Phys., 55, No. 4, 1208-1211 (1984).

16. O. I. Velichko, Mechanism of locally accelerated diffusion of impurities in high-concentration doping of silicon by phosphorus, in: Electronic Engineering, Ser. 2, Semiconducting Devices, Collection of scientific-technical papers, Issue 2, No. 187, 57-63 (1987).

17. B. J. Mulvaney and W. B. Richardson, Model for defect-impurity pair diffusion in silicon, Appl. Phys. Lett., 51, No. 18, 1439-1441 (1987).

18. M. Orlowski, Unified model for impurity diffusion in silicon, Appl. Phys. Lett., 53, No. 14, 1323-1325 (1988).

19. M. Uematsu, Simulation of boron, phosphorus, and arsenic diffusion in silicon based on an integrated diffusion model, and the anomalous phosphorus diffusion mechanism, J. Appl. Phys., 82, No. 5, 2228-2246 (1997).

20. O. I. Velichko and A. K. Fedotov, Simulation of point defect diffusion in semiconductors, Solid State Phenom., 69-70, 513-518 (1999).

21. A. K. Fedotov, O. I. Velichko, and V. A. Dobrushkin, Set of equations for stress-mediated evolution of the nonequilibrium dopant-defect system in semiconductor crystals, J. Alloys Compd., 382(1-2), 283-287 (2004).

22. B. F. Ormont, Introduction to the Physical Chemistry and Crystal Chemistry of Semiconductors [in Russian], Vysshaya Shkola, Moscow (1982).

23. H. Wagner, A. Dastgheib-Shirazi, R. Chen, et al., Improving the predictive power of modeling the emitter diffusion by fully including the phosphosilicate glass (PSG) layer, in: Proc. 37th IEEE Photovoltaic Specialists Conf. (PVSC), Seattle, USA, 19-24 June, 2011, pp. 2961-2966.

24. M. Kessler, D. Münster, T. Neubert, et al., High-efficiency back-junction silicon solar cell with an in-line evaporated aluminum front grid, in: Proc. 37th IEEE Photovoltaic Specialists Conf. (PVSC), Seattle, USA, 19-24 June, 2011, pp. 1085- 1090 .

25. L. Yang, Q. H. Ye, A. Ebong, et al., High efficiency screen printed bifacial solar cells on monocrystalline CZ silicon, Prog. Photovolt: Res. Appl., 19, 275-279 (2011).

26. S. P. Phang and D. Macdonald, Direct comparison of boron, phosphorus, and aluminum gettering of iron in crystalline silicon, J. Appl. Phys., 109, No. 073521 (2011).

27. H. Park, S. J. Tark, C. S. Kim, et al., Effect of the phosphorus gettering on Si heterojunction solar cells, Int. J. Photoenergy, 2012, Art. ID 794876 (2012).

28. O. I. Velichko and A. K. Fedotov, A model of coupled diffusion of impurity atoms and point defects in the vicinity of semiconductor interfaces and grain boundaries, Solid State Phenom., 57-58, 513-518 (1997).

29. K. Ravi, Defects and Impurities in Semiconducting Silicon [Russian translation], Mir, Moscow (1984).

30. O. I. Velichko and N. A. Sobolevskaya, Clustering of phosphorus atoms in silicon, Nonlinear Phenom. Complex Syst., 11, No. 3, 316-386 (2008).

31. R. D. Richtmyer and K. W. Morton, Difference Methods for Initial-Value Problems, Wiley and Sons, New York (1967).

32. S. Solmi, Dopants in silicon: Activation and deactivation kinetics, Encyclopedia of Materials: Science and Technology, Elsevier Science Ltd. (2001), p. 2331-2340.

33. R. B. Fair, Physics and Chemistry of Impurity Diffusion and Oxidation of Silicon. Silicon Integrated Circuits, Academic Press, New York (1981), pp. 1-56.

34. D. Mathiot and J. C. Prister, Dopant diffusion in silicon: A consistent view involving nonequilibrium defects, J. Appl. Phys., 55, No. 10, 3518-3530 (1984).

35. P. Fahey, G. Barbuscia, M. Moslehi, and R. W. Dutton, Kinetics of thermal nitridation processes in the study of dopant diffusion mechanisms in silicon, Appl. Phys. Lett., 46, No. 8, 784-786 (1985).

36. F. F. Morehead and R. F. Lever, Enhanced "tail" diffusion of phosphorus and boron in silicon: Self-interstitial phenomena, Appl. Phys. Lett., 48, No. 2, 150-151 (1986). 
37. P. B. Moynagh and P. J. Rosser, Quantification of diffusion mechanisms of boron, phosphorus, arsenic, and antimony in silicon, in: Proc. ESSDERC'89, 19th European Solid State Device Research Conf., 11-14 September, 1989, SpringerVerlag, Berlin (1989), pp. 291-296.

38. T. Shimizu, T. Takagi, S. Matsumoto, et al., Fraction of interstitialcy component of phosphorus and antimony diffusion in silicon, Jpn. J. Appl. Phys., 37, No. 3B, Pt. 1, 1184-1187 (1998).

39. O. Krause, H. Ryssel, and P. Pichler, Determination of aluminum diffusion parameters in silicon, J. Appl. Phys., 91, No. 9, 5645-5649 (2002). 